\title{
Three-Dimensional Modeling of the Heat-Affected Zone in Laser Machining Applications
}

\author{
Martynas Beresna, ${ }^{1}$ Titas Gertus, ${ }^{2,3}$ Rolandas Tomašiūnas, ${ }^{1}$ \\ Hiroaki Misawa, ${ }^{4}$ and Saulius Juodkazis ${ }^{4}$ \\ ${ }^{1}$ Institute of Materials Science and Applied Research, Sauletekio 9, 2040 Vilnius, Lithuania \\ ${ }^{2}$ Altechna Co. Ltd., Konstitucijos Avenue 23C-604, 08105 Vilnius, Lithuania \\ ${ }^{3}$ Laser Research Center, Vilnius University, Sauletekio 10, 10223 Vilnius, Lithuania \\ ${ }^{4}$ Research Institute for Electronic Science, Hokkaido University, N21W10, Kitaku, Sapporo 001-0021, Japan \\ Correspondence should be addressed to Saulius Juodkazis, saulius@es.hokudai.ac.jp \\ Received 5 May 2008; Accepted 16 September 2008 \\ Recommended by Stavros Pissadakis
}

Thermal load as well as its three-dimensional (3D) spatial distribution has been estimated inside representative materials: glass (low thermal diffusion), silicon (semimetal properties), and sapphire (a crystalline dielectric of a high thermal conductivity) for typical laser processing and direct laser writing applications. The 3D temperature distribution allows to calculate thermal stress around the focal region. This provides an assessment tool for optimization of laser microprocessing conditions for controlled laser dicing and cutting applications.

Copyright ( $) 2008$ Martynas Beresna et al. This is an open access article distributed under the Creative Commons Attribution License, which permits unrestricted use, distribution, and reproduction in any medium, provided the original work is properly cited.

\section{Introduction}

Laser processing is expanding fast into micro- and nanostructuring of complex multilayered and composite materials, heterostructures, and MEMS devices. Hence, precise account of energy delivery, heat-affected zone (HAZ), and thermal stress is of a paramount importance for high-precision fabrication and optical waveguide recording which usually have a narrow parameter space for optimization [1-9]. A high speed is required for efficient material processing by the direct laser writing $(0.1-1 \mathrm{~m} / \mathrm{s}$ for a linear scan), and it recently becomes achievable due to improvements in femtosecond laser sources which delivers large $(\sim 10 \mathrm{~W})$ average power, at $\sim \mathrm{MHz}$ repetition rate with a tens-of- $\mu$ J pulse energy.

Picosecond and femtosecond lasers are the most prospective in the nano-microdrilling, dicing, and cutting applications where micrometer precision is required. At high repetition rates, thermal effects are pronounced and are usually responsible for crack formation in the case of inbulk fabrication when nanosecond lasers are employed [10, 11]. For the waveguide recording, there are remarkable differences in defect and stress generation depending on pulse duration, scanning speed, and repetition rate $[12,13]$. In the case of shorter pulses, the size and positioning of microcracks inside the bulk of transparent materials can be achieved with high precision avoiding damage of the entrance and exit surfaces. This allows to realize a, socalled, stealth dicing when the cracks inside workpiece are large enough for successful cleaving of the sample while there is no optical damage on both surfaces after laser irradiation $[14,15]$. Similarly, cleaving of glasses can be controlled by designing a temperature field for a creation of a tensile stress which, first, forms and then guides a crack on the surface. There is a considerable interest in modeling of thermal fields inside the bulk of materials since they can be used for a controlled recording of waveguides, laser joining, and splitting applications in material processing.

Here, we explore numerically the temperature distribution inside bulk of a workpiece in different materials at realistic conditions of microprocessing by ultrafast (sub-1 ps) lasers. Combination of several laser pulses separated in time and space can be also modeled by the same approach for 
practical 3D laser dicing and writing (photo-modification) applications.

\section{Model}

Recently, experimental results on laser welding and joining of glass $[16,17]$ have been quantitatively modeled by an inbulk scanning of a rectangular heat source created at the focus of femtosecond laser beam [16]. Such a seemingly approximate description of the focal volume in the model nevertheless delivered good fit to the experimental data [16]. Hence, we use this model of the rectangular heat source for simulation of thermal field in different materials. Limitations of this approach are expected for focal spots of few micrometers in cross-section and at the very early stages of thermal diffusion, which were not considered here. It is also important that the model provides analytical solution, hence, the physical description and scaling rules of the laser processing can be qualitatively predicted for the more complex sample composition and geometry. For the quantitative modeling, however, the finite-difference timedomain (FDTD) simulations should be carried out for the actual sample geometry.

We use this model and calculate the extension of HAZ in glass, silicon, and sapphire. The dimensions of the heating source corresponds to tight focusing with numerical aperture $\mathrm{NA} \simeq 0.5$, which is a practical choice in laser microfabrication. We simulate the temperature field for a heat-source: a rectangular with a base area $(2 a)^{2}$ and height $2 h$ (in $z$-direction), scanned along $x$-direction at speed $v$ inside infinite material (Figure 1(a)). This model is realistic for actual laser processing, waveguide recording, and welding experiments when the $3 \mathrm{D}$ dimensions of the workpiece are much larger than dimensions of the heat-source (focal volume).

The temperature rise $(\theta)$ at the time moment $(t)$ at the location $(x, y, z)$ in quasi-steady state is [16]

$$
\begin{aligned}
& \theta(x, y, z, t) \\
& =\frac{A Q}{64 c_{p} \rho r} \sum_{i=0}^{\infty}\left(\operatorname{Erfc} \frac{\xi+i v \Xi^{2} / 2-\delta}{\tau \sqrt{i \Xi^{2} / \tau^{2}+1}}-\operatorname{Erfc} \frac{\xi+i v \Xi^{2} / 2+\delta}{\tau \sqrt{i \Xi^{2} / \tau^{2}+1}}\right) \\
& \times\left(\operatorname{Erfc} \frac{\psi-\delta}{\tau \sqrt{i \Xi^{2} / \tau^{2}+1}}-\operatorname{Erfc} \frac{\psi+\delta}{\tau \sqrt{i \Xi^{2} / \tau^{2}+1}}\right) \\
& \times\left(\operatorname{Erfc} \frac{\zeta-\eta}{\tau \sqrt{i \Xi^{2} / \tau^{2}+1}}-\operatorname{Erfc} \frac{\zeta+\eta}{\tau \sqrt{i \Xi^{2} / \tau^{2}+1}}\right),
\end{aligned}
$$

where $\operatorname{erfc}(U)=(2 / \sqrt{\pi}) \int_{U}^{\infty} \exp \left(-u^{2}\right) d u, A$ is absorbance of the laser pulse, $Q$ is the instantaneous laser energy, $2 a$ is the diameter of the focus, $2 h$ is the length of a heat source along $z$-axis, $c_{p}$ is specific heat, and $\rho$ is the mass density. The following nondimensional parameters are used in (1): $r^{3}=a^{2} h, \xi=x / r, \psi=y / r, \zeta=z / r, \delta=a / r=1 / \sqrt[3]{n}$, $\eta=h / r=n^{2 / 3}, \tau=\sqrt{4 \chi t} / r, \Xi=\sqrt{4 \chi T} / r$, and $v=v r /(2 \chi)$ (here $\chi$ is the temperature diffusivity).

\section{Results and Discussion}

We compare here axial temperature distributions for a highpower and high-repetition-rate heat source which models laser cutting, dicing, and waveguide recording when a focal spot is set inside the processed material. Materials with different thermal conductivity (thermal diffusivity) were modeled. The formulae given in Section 2 can be used for estimations of both axial and lateral cross-sections of the temperature field. In the following part, we present the temperature profiles along the beam scanning. The extension of lateral cross-section is closely matching that of the axial around the maximum temperature (see, Figure 1(b)). In order to compare thermal fields in very different materials, we used the same absorbance $A=0.5$ which corresponds to a strong ionization of material, for example, at the optical breakdown conditions of dielectrics such as silica or sapphire $[18,19]$, and is relevant for laser processing by ultrashort pulses at high irradiance. For more quantitative estimations, the absorbance of material and its temporal evolution during the pulse of high irradiance should be known at the focal volume.

\subsection{Temperature Field}

Figure 2 shows the thermal profiles along the scan direction with heat source at $x=0$ at different time moments within the period of laser pulsing inside glass. Similar conditions are created in glass-welding applications where high-temperature melts are essential for good quality joints $[16,17]$. The spatial extension of the heated zone does not change significantly beyond a $\pm 5 \mu \mathrm{m}$ range, only the maximum temperature is changing at the origin of the heat source. Trailing temperature distribution is recognizable at negative $x$ values.

Silicon differs from glass mainly by the thermal diffusivity which is $\chi=0.8 \mathrm{~cm}^{2} / \mathrm{s}$ (for a glass $4.6 \times 10^{-3} \mathrm{~cm}^{2} / \mathrm{s}$ ), while the specific heat and mass density are almost the same. This makes almost impossible to effectively heat and localize the hot spot inside silicon as it is shown in Figure 3. Geometrical parameters of the heat source (laser-heated focal volume) were the same as for glass at the same scanning speed of $10 \mathrm{~cm} / \mathrm{s}$. The heating power was increased 50 times to $Q=$ $10 \mathrm{~W}$ and repetition rate was 1 or $10 \mathrm{MHz}$ in order to heat up the focal volume (Figure 3 ). When repetition rate is larger (see Figure 3, curves $(2,3)$ ), there is a significant spread of temperature beyond the acceptable $\pm 5 \mu \mathrm{m}$ range. This would be critical for dicing of wafers with microelectronic chips at narrow street width. It is noteworthy that the heat capacity of solid and molten silicon is almost the same. This entitles a quantitative comparison of theoretical simulations and experiments in terms of thermal field. It should be noted that the optical properties change upon melting: the complex refractive index is changing from $n+i k=3.69+6 \times 10^{-3} i$ (crystalline) silicon to $(3.9+5.5 i)$ (liquid) silicon (for the silica native oxide, the refractive index is $1.45+4.6 \times 10^{-6} i$ ). The phase transition, the latent heat necessary to melt the focal volume, was not included in the used simple model. Hence, the obtained thermal field close to the melting point 


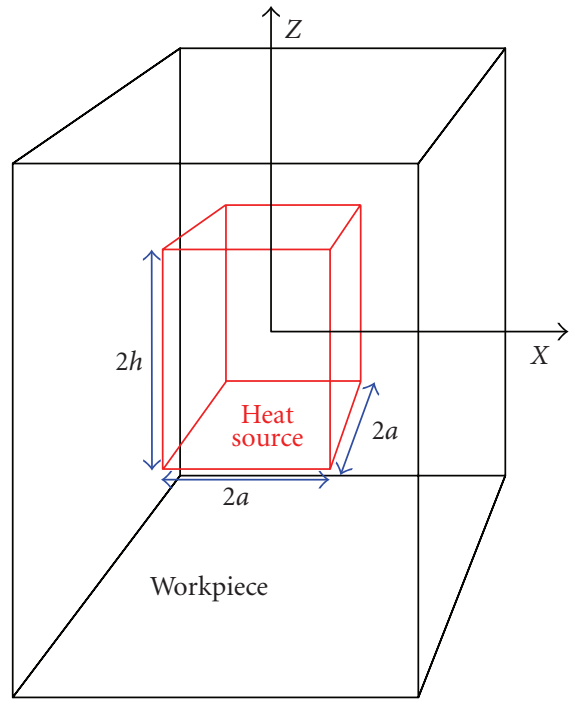

(a)

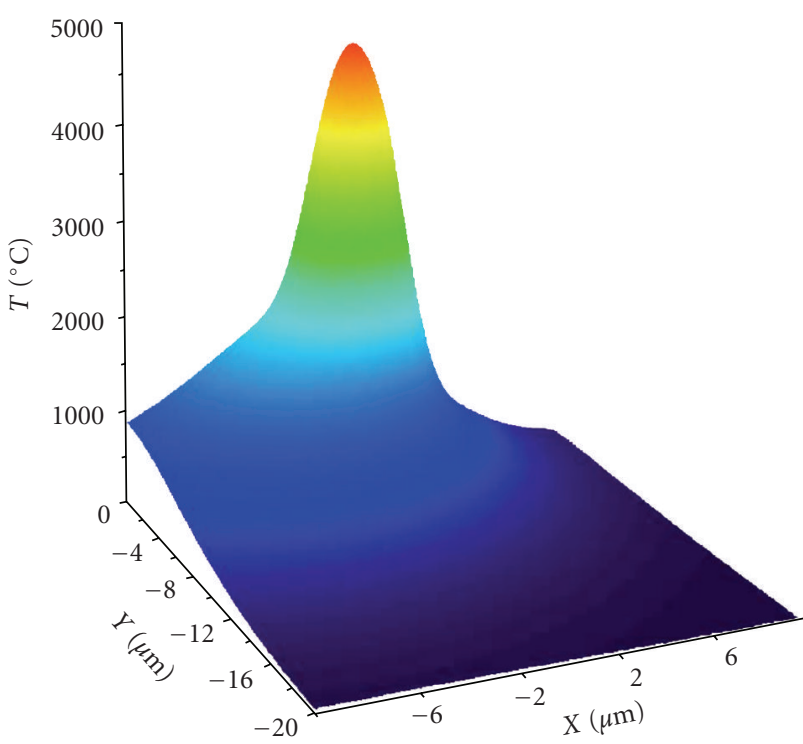

(b)

FIgURE 1: (a) Schematic presentation of the heat source with a square cross-section, $2 a \times 2 a$, on the $x y$-plane and $2 h$ high. (b) The $3 \mathrm{D}$ temperature field in glass calculated by (1) with the same parameters as in Figure 2 at time moment $t=0.1 T(T=1 / f$ where $f$ is the repetition rate) for $z=0$.

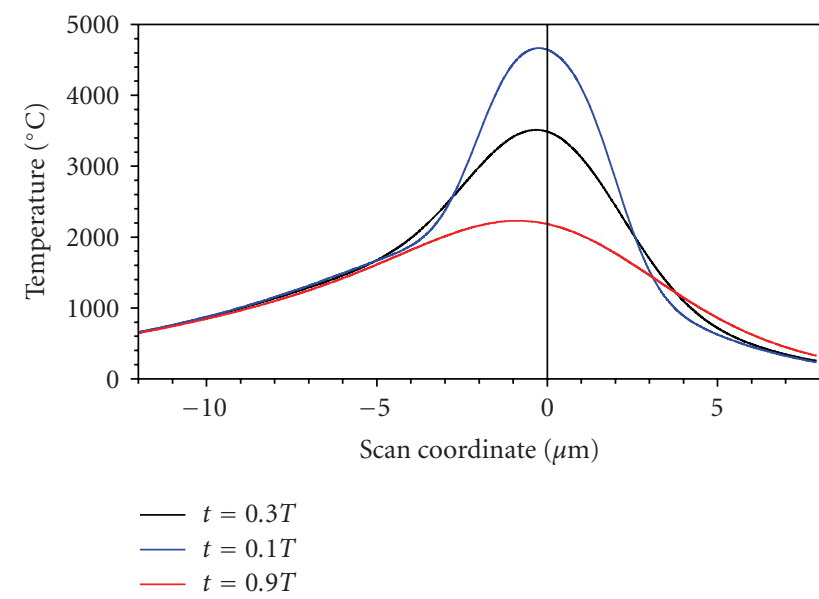

Figure 2: The axial temperature profile in glass at different time moments. Conditions: $Q=0.2 \mathrm{~W}$ at $f=0.1 \mathrm{MHz}, A=0.5$ absorbance, $10 \mathrm{~cm} / \mathrm{s}$ scan speed, the rectangular heat source is modeled by $2 \times 2 \times 20 \mu \mathrm{m}^{3}$ volume $(a \times a \times h)$. Typical borosilicate glass parameters were taken from [16].

is only approximate (Figure 3 ) since the increased absorbtion is not taken into account.

The in-bulk structuring of silicon remains a challenging task due to required high power and repetition rate; moreover, the wavelength of irradiation should be in the transparency region (a cutoff is at approximately $1.1 \mathrm{eV}$ or $1120 \mathrm{~nm}$ in Si). Typical fs-lasers are usually operating at slightly shorter wavelengths. This makes them useful only for cutting and dicing of silicon by ablation.

Sapphire stands out from transparent dielectric materials due to its high thermal conductivity (though, inferior to

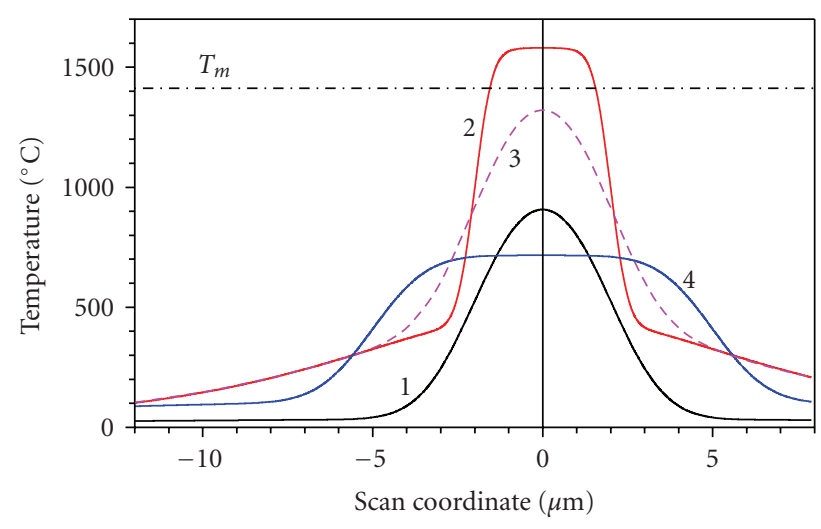

Figure 3: The axial temperature profile in Si. Conditions: $Q=$ $10 \mathrm{~W}$ at $f=1 \mathrm{MHz}$ (profiles: 1,4$)$ and $10 \mathrm{MHz}(2,3), A=0.5$ absorbance, $10 \mathrm{~cm} / \mathrm{s}$ scan speed, time moment $t=0.01 T(1,2$, 4) and $0.1 T(3)$, and dimensions of a rectangular heat source were $a=2, h=20 \mu \mathrm{m}(1-3)$, and $a=5 \mu \mathrm{m}$ (4). Typical Si parameters: $c_{p}=0.7 \mathrm{~J} /(\mathrm{g} \mathrm{K}), \rho=2.329 \mathrm{~g} / \mathrm{cm}^{3}, \chi=0.8 \mathrm{~cm}^{2} / \mathrm{s}$, and $T_{m}=1412^{\circ} \mathrm{C}$ is the melting temperature of $\mathrm{Si}$.

diamond which has a higher thermal conductivity than many metals). In terms of temperature diffusivity relevant in this study, sapphire has a $\chi=0.16 \mathrm{~cm}^{2} / \mathrm{s}$ value. Thus, it is expected to be able to localize heat more similar to silicon than glass. Figure 4 summarizes data, which show fast relaxation of maximum temperature.

The estimations given above are of qualitative nature since we are not considering here nonlinear effects of light propagation, filamentation, and self-focusing. However, general tendencies are depicted and can be used as first estimate of the thermal load to regions in close proximity of 


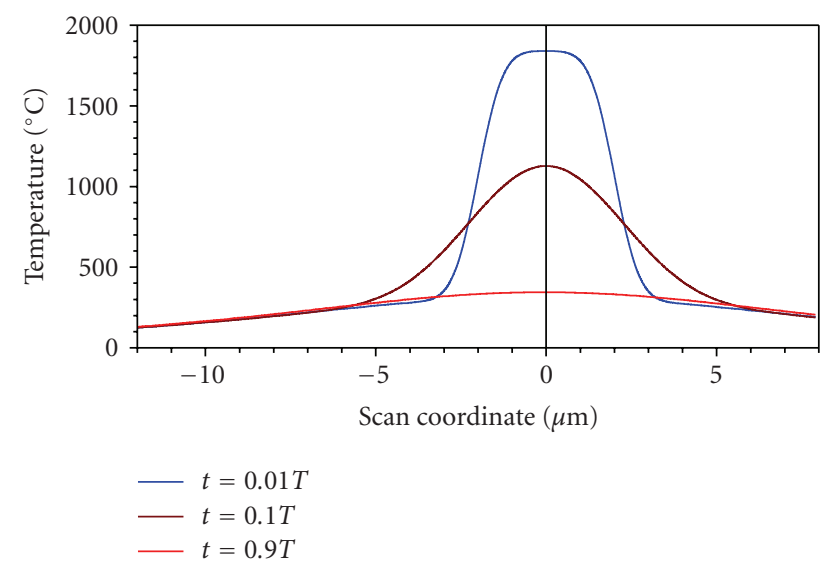

FIgURE 4: The axial temperature profile in sapphire at different time moments. Conditions: $Q=5 \mathrm{~W}$ at $f=1 \mathrm{MHz}, A=$ 0.5 absorptivity, $10 \mathrm{~cm} / \mathrm{s}$ scan speed, the rectangular heat source dimensions were $a=2, h=20 \mu \mathrm{m}$. Typical sapphire parameters [20]: $c_{p}=0.761 \mathrm{~J} /(\mathrm{g} \mathrm{K}), \rho=3.9 \mathrm{~g} / \mathrm{cm}^{3}, \chi=0.16 \mathrm{~cm}^{2} / \mathrm{s}$, and $T_{m}>2050^{\circ} \mathrm{C}$ is the melting temperature of sapphire.

the irradiated laser spot. The most challenging task in laser processing of transparent materials is the evaluation of the amount of absorbed energy and the volume of its deposition. Recent success of this model [16] in description of glass welding encourages to apply it to other laser-processing tasks such as dicing, cutting, and waveguide recording in different materials.

\subsection{Stress Confinement}

With the known thermal field calculated numerically or analytically, one can estimate the stress acting inside materials due to thermal expansion. We discuss here a model where material is described by its average thermomechanical properties, hence, the model is better suited for glasses and amorphous materials rather than crystals. However, a qualitative mechanism of the stress confinement should be valid for crystals as well. It is noteworthy that the crystalline properties become more. important in crack propagation discussed in section 3.3.

For example, for a linear heat source scanned along $x$ direction inside isotropic medium, the stress along $y$-axis is given by [14]

$$
\sigma_{y}(x)=\sigma_{\max }\left(e^{(x / l)} \mathbf{K}_{1}\left(\frac{|x|}{l}\right) \operatorname{sign} x-\frac{l}{x}\right),
$$

where $\sigma_{\max }=q \mu \alpha(1+\nu) /(2 \pi d \kappa)[\mathrm{Pa}]$ is the maximum stress for the heating power $q[\mathrm{~W}], \mathbf{K}_{1}$ is the modified Bessel function of the first kind and order, $v$ is the Poisson ratio, $l=\kappa /(c v)$ is the length of thermal diffusion expressed via the specific heat capacity density $c$ and the thermal conductivity $\kappa, v$ is the velocity of the heat source, $d$ is the thickness of sample (in this case equal to the length of the heat source). This expression (2) links the absorbed heat to the generated stress. In the case of a heating source of finite dimensions, the temperature field can be calculated by (1) and then integrated by the same way as in the case of (2) according to $[14]$

$$
\sigma_{y}(x)=\mu(1+\nu) \alpha\left[\int_{0}^{l} T(x, \xi) d \xi-T(x)\right] .
$$

It should be noted that analytical result is not available in the case of a heat source of finite dimensions and (3) should be integrated numerically.

The stress field around waveguides recorded by the femtosecond direct laser writing inside Nd-doped yttrium aluminium garnet (YAG) can be engineered to exert compressive and tensile stresses on the order of $\pm 1 \mathrm{kbar}$, respectively (up to $+5 \mathrm{kbar}$ for compression). Such photomodification can create the following: (i) waveguiding regions due to augmented refractive index $\Delta n=\left(\left(n^{2}-1\right)\left(n^{2}+2\right) / 2 n\right)(P / E)$, here $P$ is the pressure and $E$ is the Young modulus, and, (ii) the spectral properties of $\mathrm{Nd}$ transitions can be effectively modified via pressure $[21,22]$. The combined effects of refractive index and emission changes can be utilized for the creation of efficiently lasing waveguide laser-written in Nd:YAG ceramics [23].

\subsection{Crack Propagation}

Laser dicing, splitting, and cutting applications rely on a seeding of photo-modification which alters material strength and serves as a crack generator. The critical stress required for the surface or in-bulk crack formation is discussed in what follows.

The theoretical strength of material, in terms of stress, can be estimated from the work necessary to increase the atomic intraplane distance by $25 \%$ at which cleaving of a crystal occurs [24]:

$$
\sigma_{\mathrm{th}}=\sqrt{\frac{\gamma E}{a_{0}}} \simeq \frac{E}{2 \pi},
$$

where $E$ is the Young modulus, $\gamma$ is the surface energy per area, and $a_{0}$ is the atomic intraplane distance. When crack is present inside material, the stress concentration at the crack tip depends on the crack shape and size [24]:

$$
\sigma_{\max }=2 \sigma \sqrt{\frac{l_{c}}{l_{\rho}}}
$$

where $2 l_{c}$ is the length of the crack (assumed to be elliptical), $\sigma$ is the applied stress, and $l_{\rho}$ is the radius at the crack tip. Crack propagation ensues at the fracture stress when $\sigma_{\text {th }}=$ $\sigma_{\max }$ or [24]

$$
\sigma_{F}=\sqrt{\frac{2 \gamma E l_{\rho}}{3 \pi a_{0} l_{c}}} .
$$

Since the material failure (strength limit) for the tensile stress is approximately ten times lower as compared with that for a compressive load (4) scanning of two or several heat sources, the laser foci, spatially separated across (or along) the scanning direction is prospective for the controlled cleaving and dicing with a literarily zero street width. The 
absorbed laser power (the heat source) depends on the linear and nonlinear absorption at the focus, that is, on irradiance and ionization level at the focus. The cooling of the two or more spatially and temporally separated laser foci can generate tensile stresses for the controlled cleaving at the optimized conditions of laser machining.

The thermal diffusion length $L_{D}=\sqrt{\chi t}$ ( $t$ is an effective irradiation duration or pulse duration) and the absorption depth $(\sim 1 / \alpha(\lambda))$ defines a typical feature size of laser micromachining via the absorption at the wavelength $\lambda$ (linear and nonlinear absorption with the absorption coefficient $\alpha$ which is intensity-/irradiance-dependent) [25]. The minimum feature sizes of the in-bulk modification or ablation smaller than both $1 / \alpha$ and $L_{D}$ are achievable via control of processing parameters: velocity of scan, laser repetition rate, and pulse energy.

The laser heating necessary to achieve fracture strength limit in compression requires very high laser power and a high temperature gradient [19], hence, it is considered impractical for the laser processing of multilayered and multicomponent workpieces. Ultrashort pulses favor creation of a fast heating due to strong nonlinear absorption and rapid thermal quenching [18]. Both factors facilitate a stress generation. Generation of compressive and tensile stresses with a particular patterns made of several spatially (e.g., by using Gauss-Bessel beams/pulses [26]) and temporally shaped beams/pulses is expected to deliver practical solutions for the surface and in-bulk laser machining as well as for other direct laser writing applications. Since the temperature field is not symmetrical around its maximum along the scanning direction (Figure 1), it may provide an explanation to the recently observed nonreciprocity effect in femtosecond laser recording in a non-centrosymmetric lithium niobate [27].

\section{Conclusions}

The knowledge of the 3D temperature field provides a possibility to estimate stresses inside the workpiece under laser fabrication. The 3D localization of temperature inside glass, silicon, and sapphire has been calculated and discussed for practical irradiation conditions in direct laser writing/dicing/cutting applications. Strong thermal gradients can be exploited for generation of tensile stress created by several heating sources (irradiation spots/patterns) and are prospective for the controlled laser cleaving. Thermal stress sources can be created by either of the following or their combination: by a cumulative fast repetition laser irradiation, by usage of ultra-short laser pulses, or by implementation of fast laser-spot (sample) scanning. The femtosecond direct laser writing can be used for the in-bulk localization of stresses and photo-modification for photonic applications [23, 27].

\section{Acknowledgments}

Financial support by a 20070305-1 grant from "Mokslininkụ Sajungos Institutas” is highly acknowledged. S. Juodkazis is grateful to Tecdia Co., Ltd. and to the Grant-in-Aid no.
19360322 from the Ministry of Education, Science, Sports, and Culture, Japan, and to a visiting professor invitation at the Institute of Materials Science and Applied Research, Vilnius University, Lithuania.

\section{References}

[1] K. M. Davis, K. Miura, N. Sugimoto, and K. Hirao, "Writing waveguides in glass with a femtosecond laser," Optics Letters, vol. 21, no. 21, pp. 1729-1731, 1996.

[2] M. Will, S. Nolte, B. N. Chichkov, and A. Tünnermann, "Optical properties of waveguides fabricated in fused silica by femtosecond laser pulses," Applied Optics, vol. 41, no. 21, pp. 4360-4364, 2002.

[3] H. Zhang, S. M. Eaton, J. Li, and P. R. Herman, "Femtosecond laser direct writing of multiwavelength Bragg grating waveguides in glass," Optics Letters, vol. 31, no. 23, pp. 3495-3497, 2006.

[4] A. H. Nejadmalayeri and P. R. Herman, "Rapid thermal annealing in high repetition rate ultrafast laser waveguide writing in lithium niobate," Optics Express, vol. 15, no. 17, pp. 10842-10854, 2007.

[5] Z. Wang, K. Sugioka, Y. Hanada, and K. Midorikawa, "Optical waveguide fabrication and integration with a micro-mirror inside photosensitive glass by femtosecond laser direct writing," Applied Physics A, vol. 88, no. 4, pp. 699-704, 2007.

[6] S. Pissadakis, R. Böhme, and K. Zimmer, "Sub-micron periodic structuring of sapphire by laser induced backside wet etching technique," Optics Express, vol. 15, no. 4, pp. 1428$1433,2007$.

[7] S. Yliniemi, S. Honkanen, A. Ianoul, A. Laronche, and J. Albert, "Photosensitivity and volume gratings in phosphate glasses for rare-earth-doped ion-exchanged optical waveguide lasers," Journal of the Optical Society of America B, vol. 23, no. 12, pp. 2470-2478, 2006.

[8] S. Juodkazis, V. Mizeikis, S. Matsuo, K. Ueno, and H. Misawa, "Three-dimensional micro- and nano-structuring of materials by tightly focused laser radiation," Bulletin of the Chemical Society of Japan, vol. 81, no. 4, pp. 411-448, 2008.

[9] S. Juodkazis, V. Mizeikis, and H. Misawa, "Three-dimensional structuring of resists and resins by direct laser writing and holographic recording," in Photoresponsive Polymers I, vol. 213 of Advances in Polymer Science, pp. 157-206, Springer, Berlin, Germany, 2008.

[10] E. Vanagas, J.-Y. Ye, M. Li, M. Miwa, S. Juodkazis, and H. Misawa, "Analysis of stress induced by a three-dimensional recording in glass," Applied Physics A, vol. 81, no. 4, pp. 725727, 2005.

[11] S. Juodkazis, H. Misawa, E. Vanagas, and M. Li, "Thermal effects and breakdown in laser microfabrication," in Proceedings of the 4th International Congress on Laser Advanced Materials Processing (LAMP '06), pp. 6-60, Kyoto, Japan, May 2006.

[12] W. J. Reichman, D. M. Krol, L. Shah, et al., "A spectroscopic comparison of femtosecond-laser-modified fused silica using kilohertz and megahertz laser systems," Journal of Applied Physics, vol. 99, no. 12, Article ID 123112, 5 pages, 2006.

[13] M. Watanabe, S. Juodkazis, J. Nishii, S. Matsuo, and H. Misawa, "Microfabrication by a high-fluence femtosecond exposure: mechanism and applications," in Photon Processing in Microelectronics and Photonics, K. Sugioka, M. C. Gower, R. F. Haglund, et al., Eds., vol. 4637 of Proceedings of SPIE, pp. 159-168, San Jose, Calif, USA, January 2002. 
[14] S. Juodkazis, H. Misawa, and I. Maksimov, "Thermal accumulation effect in three-dimensional recording by picosecond pulses," Applied Physics Letters, vol. 85, no. 22, pp. 5239-5241, 2004.

[15] S. Juodkazis and H. Misawa, "Laser processing of sapphire by strongly focused femtosecond pulses," Applied Physics A, vol. 93, no. 4, pp. 857-861, 2008.

[16] I. Miyamoto, A. Horn, J. Gottmann, D. Wortmann, and F. Yoshino, "Fusion welding of glass using femtosecond laser pulses with high-repetition rates," Journal of Laser Micro/ Nanoengineering, vol. 2, no. 1, pp. 57-63, 2007.

[17] W. Watanabe, S. Onda, T. Tamaki, and K. Itoh, "Direct joining of glass substrates by $1 \mathrm{kHz}$ femtosecond laser pulses," Applied Physics B, vol. 87, no. 1, pp. 85-89, 2007.

[18] E. E. Gamaly, S. Juodkazis, K. Nishimura, et al., "Lasermatter interaction in the bulk of a transparent solid: confined microexplosion and void formation," Physical Review B, vol. 73, no. 21, Article ID 214101, 15 pages, 2006.

[19] S. Juodkazis, K. Nishimura, S. Tanaka, et al., "Laser-induced microexplosion confined in the bulk of a sapphire cystal: evidence of multimegabar pressures," Physical Review Letters, vol. 96, no. 16, Article ID 166101, 4 pages, 2006.

[20] S. Juodkazis, K. Nishimura, H. Misawa, et al., "Control over the crystalline state of sapphire," Advanced Materials, vol. 18, no. 11, pp. 1361-1364, 2006.

[21] S. Kobyakov, A. Kamińska, A. Suchocki, D. Galanciak, and M. Malinowski, " $\mathrm{Nd}^{3+}$-doped yttrium aluminum garnet crystal as a near-infrared pressure sensor for diamond anvil cells," Applied Physics Letters, vol. 88, no. 23, Article ID 234102, 2 pages, 2006.

[22] G. A. Torchia, P. F. Meilán, A. Rodenas, D. Jaque, C. Mendez, and L. Roso, "Femtosecond laser written surface waveguides fabricated in Nd:YAG ceramics," Optics Express, vol. 15, no. 20, pp. 13266-13271, 2007.

[23] G. A. Torchia, A. Rodenas, A. Benayas, E. Cantelar, L. Roso, and D. Jaque, "Highly efficient laser action in femtosecondwritten Nd:yttrium aluminum garnet ceramic waveguides," Applied Physics Letters, vol. 92, no. 11, Article ID 111103, 3 pages, 2008.

[24] T. H. Courtney, Mechanical Behavior of Materials, McGrawHill International Editions: Material Science/Metallurgy Series, McGraw-Hill, New York, NY, USA, 2nd edition, 2000.

[25] S. Nolte, C. Momma, H. Jacobs, et al., "Ablation of metals by ultrashort laser pulses," Journal of the Optical Society of America B, vol. 14, no. 10, pp. 2716-2722, 1997.

[26] A. Marcinkevicius, S. Juodkazis, S. Matsuo, V. Mizeikis, and H. Misawa, "Application of Bessel beams for microfabrication of dielectrics by femtosecond laser," Japanese Journal of Applied Physics, vol. 40, no. 11A, pp. L1197-L1199, 2001.

[27] W. Yang, P. G. Kazansky, and Y. P. Svirko, "Non-reciprocal ultrafast laser writing," Nature Photonics, vol. 2, no. 2, pp. 99104, 2008. 